\title{
Analysis of a Guard Condition in Type Theory (Extended Abstract)
}

\author{
Roberto M. Amadio Solange Coupet-Grimal \\ Université de Provence, Marseille *
}

\begin{abstract}
We present a realizability interpretation of co-inductive types based on partial equivalence relations (per's). We extract from the per's interpretation sound rules to type recursive definitions. These recursive definitions are needed to introduce 'infinite' and 'total' objects of coinductive type such as an infinite stream, a digital transducer, or a nonterminating process. We show that the proposed type system subsumes those studied by Coquand and Gimenez while still enjoying the basic syntactic properties of subject reduction and strong normalization with respect to a confluent rewriting system first put forward by Gimenez.
\end{abstract}

\section{Introduction}

Coquand proposes in [4] an approach to the representation of infinite objects such as streams and processes in a predicative type theory extended with coinductive types. Related analyses on the role of co-inductive types (or definitions) in logical systems can be found in $[14,11]$ for the system $F,[16]$ for the system HOL, and [20] for Beeson's Elementary theory of Operations and Numbers. Two important features of Coquand's approach are that: (1) Co-inductive types, and related constructors and destructors, are added to the theory, rather than being represented by second order types and related $\lambda$-terms, as in $[7,17]$. (2) Recursive definitions of infinite objects are restricted so that consideration of partial elements is not needed. Thus this work differs from work on the representation of infinite structures in lazy programming languages like Haskell (see, e.g., [21]).

In his thesis [8], Gimenez has carried on a realization of Coquand's programme in the framework of the calculus of constructions [5]. More precisely, he studies a calculus of constructions extended with a type of streams (i.e., finite and infinite lists), and proves subject reduction and strong normalization for a related confluent rewriting system. He also applies co-inductive types to the representation and mechanical verification of concurrent systems by relying on the Coq system [3] extended with co-inductive types (another case study can be found in [6]). In this system, processes can be directly represented in the logic as elements of a certain type. This approach differs sharply from those where, say,

\footnotetext{
* CMI, 39 rue Joliot-Curie F-13453, Marseille, France. amadio@gyptis.univ-mrs.fr. The first author was partially supported by CTI-CNET 95-1B-182, Action Incitative INRIA, IFCPAR 1502-1,WG Confer, and HCM Express. A preliminary version of this paper (including proofs) can be found in [1].
} 
processes are represented at a syntactic level as elements of an inductively defined type (see, e.g., [15]). Clearly the representation based on co-inductive types is more direct because recursion is built-in. This may be a decisive advantage when carrying on formal proofs. Therefore, the issue is whether this representation is flexible enough, that is whether we can type enough objects and whether we can reason about their equality. These questions are solid motivations for our work.

The introduction of infinite 'total' objects relies on recursive definitions which are intuitively 'guarded' in a sense frequently arising in formal languages [18]. An instance of the new typing rule in this approach is:

$$
\frac{\Gamma, x: \sigma \vdash M: \sigma M \downarrow x \sigma \text { co-inductive type }}{\Gamma \vdash \text { fix } x \cdot M: \sigma} .
$$

This allows for the introduction of 'infinite objects' in a 'co-inductive type', by means of a 'guarded' (recursive) definition. Of course, one would like to have notions of co-inductive type and of guarded definition which are as liberal as possible and that are supported by an intuitive, i.e., semantic, interpretation.

In Coquand's proposal, the predicate $M+x$ is defined by a straightforward analysis of the syntactic structure of the term. This is a syntactic approximation of the main issue, that is to know when the recursive definition fix $x . M$ determines a unique total object. To answer this question we interpret co-inductive types in the category of per's (partial equivalence relations), a category of total computations, and we find that the guard predicate $M \downarrow x$ has a semantic analogy which can be stated as follows:

$$
\forall \alpha\left((d, e) \in \mathcal{F}_{\sigma}^{\alpha} \Rightarrow\left(\llbracket M \rrbracket[d / x], \llbracket[M \rrbracket[e / x]) \in \mathcal{F}_{\sigma}^{\alpha+1}\right)\right.
$$

where $\mathcal{F}_{\sigma}$ is a monotonic function on per's associated to the co-inductive type $\sigma$, and $\mathcal{F}_{\sigma}^{\alpha}$ is its $\alpha^{\text {th }}$ iteration, for $\alpha$ ordinal. We propose to represent condition (2) in the syntax by introducing some extra-notation. With the side conditions of rule (1), we introduce two types $\dot{\sigma}$ and $\tilde{\sigma}^{+}$which are interpreted respectively by $\mathcal{F}_{\sigma}^{\alpha}$ and $\mathcal{F}_{\sigma}^{\alpha+1}$. We can then replace the guard condition $M \downarrow x$ by the typing judgment $x: \check{\sigma} \vdash M: \check{\sigma}^{+}$whose interpretation is basically condition (2). The revised typing system also includes: (1) Subtyping rules which relate a co-inductive type $\sigma$ to its approximations $\check{\sigma}$ and $\check{\sigma}^{+}$, so that we will have: $\sigma \leq \tilde{\sigma}^{+} \leq \dot{\sigma}$. (2) Rules which overload the constructors of the co-inductive type, e.g., if $f: \sigma \rightarrow \sigma$ is a unary constructor over $\sigma$, then $f$ will also have the type $\check{\sigma} \rightarrow \check{\sigma}^{+}$(to be understood as $\forall \alpha x \in \mathcal{F}_{\sigma}^{\alpha} \Rightarrow f(x) \in \mathcal{F}_{\sigma}^{\alpha+1}$ ). The types $\sigma \rightarrow \sigma$ and $\check{\sigma} \rightarrow \check{\sigma}^{+}$will be incomparable with respect to the subtyping relation.

The idea of expressing the guard condition via approximating types, subtyping, and overloading can be traced back to Gimenez's system. Our contribution here is to provide a semantic framework which:

(1) Justifies and provides an intuition for the typing rules. In particular, we will see how it is possible to understand semantically Gimenez's system.

(2) Suggests new typing rules and simplifications of existing ones. In particular, we propose: (i) a rule to type nested recursive definitions, and (ii) a way to type recursive definitions without labelling types.

(3) Can be readily adapted to prove strong normalization with respect to the confluent reduction relation introduced by Gimenez. 


\section{A simply typed calculus}

We will carry on our study in a simply typed $\lambda$-calculus extended with coinductive types. ${ }^{2}$ Let $F$ be a countable set of constructors. We let $f_{1}, f_{2}, \ldots$ range over $F$. Let $t v$ be the set of type variables $t, s, \ldots$ The language of raw types is given by the following (informal) grammar:

$$
\tau::=t v\left|\left(\tau \rightarrow \tau^{\prime}\right)\right| \nu t v \cdot\left(f_{1}: \tau_{1} \rightarrow t v \ldots f_{k}: \tau_{k} \rightarrow t v\right)
$$

where $\tau_{i} \rightarrow t v$ stands for $\tau_{i, 1} \rightarrow \cdots \rightarrow \tau_{i, n_{i}} \rightarrow t v(\rightarrow$ associates to the right), and all $f_{i}$ are distinct. Intuitively, a type of the shape $\nu t .\left(f_{1}: \tau_{1} \rightarrow t \ldots f_{k}: \tau_{k} \rightarrow t\right)$ is well-formed if the type variable $t$ occurs positively in the well-formed types $\tau_{i, j}$, for $i=1 \ldots k, j=1 \ldots n_{i}$. Note that the type variable $t$ is bound by $\nu$ and it can be renamed. We call types of this shape co-inductive types, the symbols $f_{1} \ldots f_{k}$ represent the constructors of the type. We will denote co-inductive types with the letters $\sigma, \sigma^{\prime}, \sigma_{1}, \ldots$, and unless specified otherwise, we will suppose that they have the generic form in (3). A precise definition of the well-formed types is given as follows.

Definition 1 types. If $\tau$ is a raw type and $s$ is a type variable then the predicates $w f(\tau)$ (well-formed), $\operatorname{pos}(s, \tau)$ (positive occurrence only), and $n e g(s, \tau)$ (negative occurrence only) are the least predicates which satisfy the following conditions.

(1) If $t \in t v$ then $w f(t)$, pos $(s, t)$, and $n e g(s, t)$ provided $t \neq s$.

(2) If $w f(\tau)$ and $w f\left(\tau^{\prime}\right)$ then $w f\left(\tau \rightarrow \tau^{\prime}\right)$. Moreover, $\operatorname{pos}\left(s, \tau \rightarrow \tau^{\prime}\right)$ if $\operatorname{pos}\left(s, \tau^{\prime}\right)$ and $n e g(s, \tau)$, and $n e g\left(s, \tau \rightarrow \tau^{\prime}\right)$ if $n e g\left(s, \tau^{\prime}\right)$ and $\operatorname{pos}(s, \tau)$.

(3) If $\sigma=\nu t .\left(f_{1}: \tau_{1} \rightarrow t \ldots f_{k}: \tau_{k} \rightarrow t\right)$ and $t \neq s$ (otherwise rename $t$ ) then $w f(\sigma)$ provided $w f\left(\tau_{i, j}\right)$ and $\operatorname{pos}\left(t, \tau_{i, j}\right)$ for $i=1 \ldots k, j=1 \ldots n_{i}$. Moreover, $\operatorname{pos}(s, \sigma)$ if $\operatorname{pos}\left(s, \tau_{i, j}\right)$ for $i=1 \ldots k, j=1 \ldots n_{i}$, and $n e g(s, \sigma)$ if $n e g\left(s, \tau_{i, j}\right)$ for $i=1 \ldots k, j=1 \ldots n_{i}$.

Example 1. Here are a few examples of well-formed co-inductive types where we suppose that the type $\tau$ is not bound by $\nu$.

(1) Infinite streams over $\tau: \nu s$.(cons: $\tau \rightarrow(s \rightarrow s)$ ).

(2) Input-output processes over $\tau: \nu p$.(nil : $p, !: \tau \rightarrow p \rightarrow p$, ?: $(\tau \rightarrow p) \rightarrow p)$.

(3) An involution: $\nu t$.(inv : $((t \rightarrow \tau) \rightarrow \tau) \rightarrow t)$.

Definition 1 allows mutually recursive definitions. For instance, we can define processes over streams over processes ...:

$$
\sigma=\nu t .\left(\text { nil }: t, !: \sigma^{\prime} \rightarrow t \rightarrow t, ?:\left(\sigma^{\prime} \rightarrow t\right) \rightarrow t\right) \quad \sigma^{\prime}=\nu s .(\text { cons }: t \rightarrow s \rightarrow s) .
$$

\footnotetext{
${ }^{2}$ Per's interpretations support other relevant extensions of the type theory, including second-order types (see, e.g., [13]) and inductive types (see, e.g., [12]). As expected, an inductive type, e.g., $\mu t$. (nil : $t$, cons : $0 \rightarrow t \rightarrow t$ ) is interpreted as the least fixpoint of the operator $\mathcal{F}$ described in section 3 . It follows that there is a natural subtyping relation between the inductive type and the corresponding co-inductive type $\nu t$.(nil : $t$, cons : $o \rightarrow t \rightarrow t$ ).
} 
These mutually recursive definitions lead to some complication in the typing of constructors. For instance, the type of cons should be $[\sigma / t]\left(t \rightarrow \sigma^{\prime} \rightarrow \sigma^{\prime}\right)$, and moreover we have to make sure that all occurrences of a cons have the same type (after unfolding). To make our analysis clearer, we prefer to gloss over these technical issues by taking a stronger definition of positivity. Thus, in the case (3) of definition 1, we say pos $(s, \sigma)$ (or $n e g(s, \sigma)$ ) if $s$ does not occur free in $\sigma$. In this way a type variable which is free in a co-inductive type cannot be bound by a $\nu$.

Let $v$ be the set of term variables $x, y, \ldots$ A context $\Gamma$ is a possibly empty list $x_{1}: \tau_{1} \ldots x_{n}: \tau_{n}$ where all $x_{i}$ are distinct. Raw terms are defined by the following grammar:

$$
M::=v|(\lambda v . M)|(M M)\left|f^{\sigma}\right| \text { case }^{\sigma} \mid(\text { fix } v . M) .
$$

We denote with $F V(M)$ the set of variables occurring free in the term $M$. The typing rules are defined as follows:

$$
\begin{gathered}
\frac{x: \tau \in \Gamma}{\Gamma \vdash x: \tau} \frac{\Gamma, x: \tau \vdash M: \tau^{\prime}}{\Gamma \vdash \lambda x \cdot M: \tau \rightarrow \tau^{\prime}} \frac{\Gamma \vdash M: \tau^{\prime} \rightarrow \tau \Gamma N: \tau^{\prime}}{\Gamma \vdash M N: \tau} \\
\text { Assuming: } \begin{array}{c}
\sigma=\nu t .\left(f_{1}: \tau_{1} \rightarrow t \ldots f_{k}: \tau_{k} \rightarrow t\right) \\
\tau^{\prime} \rightarrow \sigma=\tau_{1}^{\prime} \rightarrow \ldots \tau_{m}^{\prime} \rightarrow \sigma(m \geq 0)
\end{array} \\
\frac{\Gamma \vdash f_{i}^{\sigma}:[\sigma / t] \tau_{i, 1} \rightarrow \cdots[\sigma / t] \tau_{i, n_{i}} \rightarrow \sigma}{\Gamma \vdash \operatorname{case}^{\sigma}: \sigma \rightarrow\left([\sigma / t] \tau_{1} \rightarrow \tau\right) \rightarrow \cdots\left([\sigma / t] \tau_{\mathbf{k}} \rightarrow \tau\right) \rightarrow \tau} \\
\frac{\Gamma, x: \tau^{\prime} \rightarrow \sigma \vdash M: \tau^{\prime} \rightarrow \sigma M+x}{\Gamma \vdash \text { fix } x . M: \tau^{\prime} \rightarrow \sigma}
\end{gathered}
$$

The guard predicate ' $M \downarrow x$ ' is left unspecified. Intuitively, this predicate has to guarantee that a recursive definition does determine a unique 'total' object. Before trying a formal definition, we will consider a few examples of recursive definitions, where we use the notation let $x=M$ in $N$ for $(\lambda x . M) N$, and let application associate to the left.

Example 2. Let $o$ be a basic type of numerals with constants $0: o$ and suc $: o \rightarrow o$. Let us first consider the type of infinite streams of numerals, with destructors head and tail:

$$
\begin{gathered}
\sigma_{1}=\nu t .(\text { cons }: o \rightarrow(t \rightarrow t)) \\
h d=\lambda x \cdot \text { case }^{\sigma_{1}} x(\lambda n \cdot \lambda y \cdot n) \quad t l=\lambda x \cdot \text { case }^{\sigma_{1}} x(\lambda n \cdot \lambda y \cdot y) .
\end{gathered}
$$

(1) We can introduce an infinite list of 0 's as follows: fix $x$.cons ${ }^{\sigma_{1}} 0 x$.

(2) We can also define a function which adds 1 to every element of a stream:

$$
\text { fix add1 } . \lambda x \cdot \text { case }^{\sigma_{1}} x\left(\lambda n \cdot \lambda x^{\prime} \cdot \operatorname{cons}^{\sigma_{1}}(\text { suc n })\left(a d d 1 x^{\prime}\right)\right) \text {. }
$$


(3) Certain recursive definitions should not type, e.g., fix $x . \operatorname{cons}^{\sigma_{1}} 0(t l x)$. The equation does not determine a stream, as all streams of the form cons ${ }^{\sigma_{1}} 0 z^{\prime}$ give a solution.

(4) The function $d b$ doubles every element in the stream:

$$
\text { fix } d b . \lambda x \text {. let } n=(h d x) \text { in } \text { cons }^{\sigma_{1}} n\left(\text { cons }^{\sigma_{1}} n d b(t l x)\right) \text {. }
$$

(5) Next we work over the type $\sigma_{2}$ of finite and infinite streams. The function $C$ concatenates two streams.

$$
\begin{aligned}
& \sigma_{2}=\nu t .(\text { nil }: t \text {, cons : } o \rightarrow t \rightarrow t) \\
& \left.C \equiv \text { fix conc. } \lambda x \cdot \lambda y . \text { case }^{\sigma_{2}} x \text { y } \lambda n . \lambda x^{\prime} \text {. (cons }{ }^{\sigma_{2}} n\left(\operatorname{con} c x^{\prime} y\right)\right) \text {. }
\end{aligned}
$$

(6) Finally, we consider the type $\sigma_{3}$ of infinite binary trees whose nodes may have two colours, and the following recursive definition:

$$
\begin{aligned}
\sigma_{3}= & \nu t .\left(\operatorname{bin}_{1}: t \rightarrow t \rightarrow t, \operatorname{bin}_{2}: t \rightarrow t \rightarrow t\right) \\
& \left(\text { fix } x . \mathrm{bin}_{1}^{\sigma_{3}} x\left(\text { fix } y \cdot \operatorname{bin}_{2}^{\sigma_{3}} x y\right)\right) .
\end{aligned}
$$

We recall next Coquand's definition [4] of the guard predicate in the case the type theory includes just one co-inductive type, say $\sigma=\nu t$.(nil : $t$, cons : $o \rightarrow$ $t \rightarrow t)$.

Definition 2. Supposing $\Gamma, x: \boldsymbol{r}^{\prime} \rightarrow \sigma \vdash M: \tau^{\prime} \rightarrow \sigma$, we write $M \downarrow x$ if the judgment $\Gamma, x: \tau^{\prime} \rightarrow \sigma \vdash M \downarrow_{1}^{\tau^{\prime} \rightarrow \sigma} x$ can be derived by the following rules, where $n$ ranges over $\{0,1\}$. The intuition is that ' $x$ is guarded by at least a constructor in $M^{\prime}$ '. For the sake of readability, we omit in the premisses the conditions that $x: \tau^{\prime} \rightarrow \sigma \in \Gamma$ and the terms have the right type.

$$
\begin{array}{cc}
\frac{x \notin F V(M)}{\Gamma \vdash M \downarrow_{n}^{\tau} x} & \frac{\Gamma, y: \tau \vdash M \downarrow_{n}^{\tau \rightarrow \sigma} x y \neq x}{\Gamma \vdash \lambda y \cdot M \downarrow_{n}^{\tau \rightarrow \tau+\sigma} x} \\
\frac{x \notin F V\left(M_{2}\right) \Gamma \vdash M_{2} \downarrow_{0}^{\sigma} x}{\Gamma \vdash \operatorname{cons}^{\sigma} M_{1} M_{2} \downarrow_{1}^{\sigma} x} & \frac{x \notin F V\left(M_{1}\right) \Gamma \vdash M_{2} \downarrow_{0}^{\sigma} x}{\Gamma \vdash \operatorname{cons}^{\sigma} M_{1} M_{2} \downarrow_{0}^{\sigma} x} \\
\frac{x \notin F V(N) \Gamma \vdash M_{1} \downarrow_{n}^{\sigma} x \Gamma \vdash M_{2} \downarrow_{n}^{o \rightarrow \sigma \rightarrow \sigma} x}{\Gamma \vdash \operatorname{case}^{\sigma} N M_{1} M_{2} \downarrow_{n}^{\sigma} x} & \frac{x \notin F V\left(M_{j}\right) j=1 \ldots m}{x M_{1} \ldots M_{m} \downarrow_{0}^{\sigma} x} .
\end{array}
$$

Coquand's definition is quite restrictive. In particular: (i) it is unable to traverse $\beta$-redexes as in example 2(4), and (ii) it does not cope with nested recursive definitions as in example 2(6). We present in the next section a simple semantic framework which clarifies the typing issues and suggests a guard condition more powerful than the one above.

\section{Interpretation}

In this section we present an interpretation of the calculus in the well-known category of partial equivalence relations (per's) over a $\lambda$-model (cf., e.g., [19]). Let $(D, \cdot, k, s, \epsilon)$ be a $\lambda \beta$-model (cf. [2]). We often write de for $d \cdot e$. We denote with $A, B, \ldots$ binary relations over $D$. We write $d A e$ for $(d, e) \in A$ and we set: $[d]_{A}=\{e \in D \mid d A e\},|A|=\{d \in D \mid d A d\}$, and $[A]=\left\{[d]_{A}|d \in| A \mid\right\}$. 
Definition 3 partial equivalence relations. Let $D$ be a $\lambda$-model. The category of per's over $D\left(\right.$ per $\left._{D}\right)$ is defined as follows:

$$
\begin{aligned}
& \operatorname{per}_{D} \quad=\{A \mid A \subseteq D \times D \text { and } A \text { is symmetric and transitive }\} \\
& \operatorname{per}_{D}[A, B]=\{f:[A] \rightarrow[B] \mid \exists \phi \in D(\phi \mid 1-f)\} \\
& \phi \mid-f:[A] \rightarrow[B] \text { iff } \forall d \in D\left(d \in|A| \Rightarrow \phi d \in f\left([d]_{A}\right)\right) .
\end{aligned}
$$

We will use the $\lambda$-notation to denote elements of the $\lambda$-model $D$. E.g., $\lambda x . x \|-f$ stands for $\llbracket \lambda x . x \rrbracket^{D} \Vdash \mid f$. The category $\operatorname{per}_{D}$ has a rich structure, in particular it has finite products, finite sums, and exponents, whose construction is recalled below.

$$
\begin{aligned}
& d A_{1} \times \ldots \times A_{n} e \text { iff } \forall i \in\{1 \ldots n\}\left(p_{i} d\right) A_{i}\left(p_{i} e\right) \text { where: } \\
& p_{i}=\lambda u . u\left(\lambda x_{1} \ldots \lambda x_{n} . x_{i}\right) \quad p_{i} \| \pi_{i}:\left[\Pi_{i=1 \ldots n} A_{i}\right] \rightarrow\left[A_{i}\right] \\
& \phi_{i} \| f_{i}:[C] \rightarrow\left[A_{i}\right] \Rightarrow \lambda d . \lambda u . u\left(\phi_{1} d\right) \ldots\left(\phi_{n} d\right) \Vdash\left\langle f_{1} \ldots f_{n}\right\rangle:[C] \rightarrow\left[\Pi_{i=1 \ldots n} A_{i}\right] \\
& d A_{1}+\ldots+A_{n} e \text { iff } \exists i \in\{1 \ldots n\} \quad\left(d=\left(j_{i} d^{\prime}\right),\left(e=j_{i} e^{\prime}\right) \text { and } d^{\prime} A_{i} e^{\prime}\right) \text { where: } \\
& j_{i}=\lambda u . \lambda y_{1} \ldots \lambda y_{n} . y_{i} u \quad j_{i} \|-i n_{i}:\left[A_{i}\right] \rightarrow\left[\Sigma_{i=1 \ldots n} A_{i}\right] \\
& \phi_{i} \Vdash \vdash f_{i}:\left[A_{i}\right] \rightarrow[C] \Rightarrow \lambda d . d \phi_{1} \ldots \phi_{n} \|-\left[f_{1} \ldots f_{n}\right]:\left[\Sigma_{i=1 . . n} A_{i}\right] \rightarrow[C] \\
& d A \rightarrow B e \text { iff } \forall d^{\prime}, e^{\prime}\left(d^{\prime} A e^{\prime} \Rightarrow\left(d d^{\prime}\right) B\left(e e^{\prime}\right)\right) \text { where: } \\
& \lambda d .\left(p_{1} d\right)\left(p_{2} d\right) \Vdash-e v:\left[B^{A} \times A\right] \rightarrow B \\
& \phi \Vdash \mid-f:[C \times A] \rightarrow[B] \Rightarrow \lambda d . \lambda d^{\prime} . \phi\left(\lambda u .(u d) d^{\prime}\right) \Vdash \Lambda(f):[C] \rightarrow\left[B^{A}\right] \text {. }
\end{aligned}
$$

As degenerate cases of empty product and empty sum we get terminal and initial objects:

$$
1=D \times D \quad \lambda x . x\|f:[A] \rightarrow 1 \quad 0=\emptyset \quad \lambda x . x\| f:[0] \rightarrow[A] .
$$

We denote with $\eta: t v \rightarrow \operatorname{per}_{D}$ type environments. The interpretation of type variables and higher types is then given as follows:

$$
\llbracket t \rrbracket_{\eta}=\eta(t) \quad \llbracket \tau \rightarrow \tau^{\prime} \rrbracket_{\eta}=\llbracket \tau \rrbracket_{\eta} \rightarrow \llbracket \tau^{\prime} \rrbracket_{\eta} .
$$

As for co-inductive types, given a type $\sigma=\nu t .\left(f_{1}: \tau_{1} \rightarrow t \ldots f_{k}: \tau_{k} \rightarrow t\right)$, and a type environment $\eta$, we define a function $\mathcal{F}_{\sigma, \eta}$ on $\operatorname{per}_{D}$ as follows:

$$
\mathcal{F}_{\sigma, \eta}(A)=\Sigma_{i=1 \ldots k}\left(\Pi_{j=1 \ldots n_{i}}\left[\tau_{i, j} \rrbracket_{\eta[A / t}\right) .\right.
$$

We then observe that per $_{D}$ is a complete lattice with respect to set-inclusion, and that thanks to the positivity condition in the definition of co-inductive type, $\mathcal{F}_{\sigma, \eta}$ is monotonic on $\operatorname{per}_{D}$. Therefore we can define ( $g f p$ stands for greatest fixpoint):

$$
\llbracket \sigma \rrbracket_{\eta}=\bigcup\left\{A \mid A \subseteq \mathcal{F}_{\sigma, \eta}(A)\right\} \quad\left(=g f p\left(\mathcal{F}_{\sigma, \eta}\right)\right) .
$$

In general, if $f$ is a monotonic function over a poset with greatest element $T$ and glb's, we define the iteration $f^{\alpha}$, for $\alpha$ ordinal as follows:

$$
f^{0}=\top \quad f^{\alpha+1}=f\left(f^{\alpha}\right) \quad f^{\lambda}=\bigwedge_{\alpha<\lambda} f^{\alpha} \quad(\lambda \text { limit ordinal }) .
$$


With this notation, we have $g f p\left(\mathcal{F}_{\sigma, \eta}\right)=\mathcal{F}_{\sigma, \eta}^{\alpha}$ for some ordinal $\alpha$.

Since $\operatorname{per}_{D}$ is a CCC there is a canonical interpretation of the simply typed $\lambda$-calculus. The interpretation of constructors and case is driven by equation (5). Note that to validate the typing rules it is enough to know that the interpretation of a co-inductive type is $a$ fixpoint of the related functional defined by equation (5) (as a matter of fact, these rules are sound also for inductive types). The interpretation of fix is more problematic (and represents the original contribution of this section as far as semantics is concerned). We proceed as follows:

- We define an erasure function er from the terms in the language to (pure) untyped $\lambda$-terms, and we interpret the untyped $\lambda$-terms in the $\lambda$-model $D$. This interpretation, is always well-defined as the $\lambda$-model accommodates arbitrary recursive definitions.

- We see what it takes for the interpretation of (the erasure of) a fixpoint to be in the corresponding type interpretation, and we derive a suitable guard condition which is expressed by additional typing rules in a suitably enriched language.

- We prove soundness of the interpretation with respect to the enriched typing system.

Definition 4 erasure. We define an erasure function from terms to (pure) untyped $\lambda$-terms, by induction on the structure of the term (assuming $\sigma=\nu t$. $\left(f_{1}\right.$ : $\left.\tau_{1} \rightarrow t \ldots f_{k}: \tau_{k} \rightarrow t\right)$ )

$$
\begin{gathered}
\operatorname{er}(x)=x \quad \operatorname{er}(\lambda x . M)=\lambda x \cdot \operatorname{er}(M) \quad \operatorname{er}(M N)=\operatorname{er}(M) \operatorname{er}(N) \\
\operatorname{er}\left(f_{i}^{\sigma}\right)=\lambda x_{1} \ldots \lambda x_{n_{i}} \cdot \lambda y_{1} \ldots \lambda y_{k} \cdot y_{i}\left(\lambda u \cdot u x_{1} \ldots x_{n_{i}}\right) \\
\operatorname{er}\left(\text { case }^{\sigma}\right)=\lambda x \cdot \lambda y_{1} \ldots \lambda y_{k} . x U\left(y_{1}\right) \ldots U\left(y_{k}\right) \text { with } U\left(y_{i}\right)=\lambda u \cdot y_{i}\left(p_{1} u\right) \ldots\left(p_{n_{i}} u\right) \\
\operatorname{er}(\text { fix } x . M)=Y(\lambda x \cdot \operatorname{er}(M)) \text { with } Y=\lambda f \cdot(\lambda x \cdot f(x x))(\lambda x \cdot f(x x)) .
\end{gathered}
$$

If $n_{i}=0$ then we have $\operatorname{er}\left(\mathrm{f}_{\mathrm{i}}{ }^{\circ}\right)=\lambda y_{1} \ldots \lambda y_{k} \cdot y_{i}(\lambda u . u)$ and $U\left(y_{i}\right)=\lambda u . y_{i}$. If $k=1$ then the definitions simplify to $\operatorname{er}\left(f_{1}{ }^{\sigma}\right)=\lambda x_{1} \ldots \lambda x_{n_{1}} \cdot \lambda u . u x_{1} \ldots x_{n_{1}}$ and $e r\left(\right.$ case $\left.^{\sigma}\right)=\lambda x \cdot \lambda y_{1} \cdot y_{1}\left(p_{1} x\right) \ldots\left(p_{n_{1}} x\right)$.

The erasures of $f_{i}{ }^{\circ}$ and case ${ }^{\sigma}$ are designed to fit the per interpretation of coinductive types, in particular they rely on the definition of sum and product in $\operatorname{per}_{D}$.

We sketch with an informal notation an instance of our semantic analysis. We write $\vDash P: \tau$ if $\llbracket P \rrbracket^{D} \in \|[\tau \rrbracket \mid$. The typing rule for recursive definitions is sound if we can establish:

$$
\vDash Y(\lambda x \cdot \operatorname{er}(M)): \sigma \text {. }
$$

Given the iterative definitions of the interpretation of the co-inductive type $\sigma$, we can try to prove:

$$
\forall \alpha \text { ordinal } \models Y(\lambda x \cdot \operatorname{er}(M)): \mathcal{F}_{\sigma}^{\alpha}
$$


by induction on the ordinal $\alpha$. The case $\alpha=0$ is trivial since $\mathcal{F}_{\sigma}^{\alpha}=1$, and the case $\alpha$ limit ordinal follows by an exchange of universal quantifications. For the case $\alpha=\alpha^{\prime}+1$, it would be enough to know:

$$
\forall \alpha\left(\vDash Y(\lambda x \cdot \operatorname{er}(M)): \mathcal{F}_{\sigma}^{\alpha} \Rightarrow \vDash Y(\lambda x \cdot \operatorname{er}(M)): \mathcal{F}_{\sigma}^{\alpha+1}\right) .
$$

Since $Y(\lambda x \cdot \operatorname{er}(M))=[Y(\lambda x \cdot \operatorname{er}(M)) / x] M$, property (9) is implied by the following property:

$$
\forall \alpha, P\left(=P: \mathcal{F}_{\sigma}^{\alpha} \Rightarrow \vDash=[P / x] \operatorname{er}(M): \mathcal{F}_{\sigma}^{\alpha+1}\right) .
$$

In order to represent this condition in the syntax, we parameterize the type interpretation on an ordinal $\alpha$, and we introduce types $\check{\sigma}$ and $\dot{\sigma}^{+}$so that $[\tilde{\sigma}]^{\alpha}=$ $\mathcal{F}_{\sigma}^{\alpha}$, and $\left[\tilde{\sigma}^{+}\right]^{\alpha}=\mathcal{F}_{\sigma}^{\alpha+1}$. Property (10) is then expressed by the judgment $x$ : $\check{\sigma} \vdash M: \check{\sigma}^{+}$.

Let $T$ be the set of types specified in definition 1 . We define the set $T^{\prime}$ as the least set such that: (i) $T \subseteq T^{\prime}$, (ii) if $\sigma \in T$ is a co-inductive type then $\check{\sigma} \in T^{\prime}$ and $\dot{\sigma}^{+} \in T^{\prime}$, and (iii) if $\tau \in T^{\prime}$ and $r^{\prime} \in T^{\prime}$ then $r \rightarrow \tau^{\prime} \in T^{\prime}$. We also define the set $T^{+}$as the set of types in $T^{\prime}$ such that all types of the form $\check{\sigma}$ and $\check{\sigma}^{+}$appear in positive position (the interpretation of these types is going to be anti-monotonic in the ordinal). If $\Gamma$ is a context then $T(\Gamma)=\{\tau \mid x: \tau \in \Gamma\}$.

The revised typing system contains the typing rules presented in section 2 (applied with the enriched set of types) but for the rule for fix which is replaced by the rules displayed below. Of course, all the rules are applied on the enriched set of types, and under the hypothesis that all types are well-formed.

$$
\begin{aligned}
& \text { Assuming: } \sigma=\nu t .\left(\mathrm{f}_{1}: \tau_{1} \rightarrow t \ldots \mathrm{f}_{\mathrm{k}}: \tau_{k} \rightarrow t\right) \\
& \tau^{\prime} \rightarrow \sigma=\tau_{1}^{\prime} \rightarrow \ldots T_{m}^{\prime} \rightarrow \sigma(m \geq 0) \\
& T(\Gamma) \cup\left\{\tau_{1}^{\prime} \ldots \tau_{m}^{\prime}\right\} \subseteq T \\
& \Gamma, x: \tau^{\prime} \rightarrow \sigma \vdash M: \tau^{\prime} \rightarrow \sigma \\
& \frac{\Gamma, x: \tau^{\prime} \rightarrow \bar{\sigma}+M: \tau^{\prime} \rightarrow \sigma^{+}}{\Gamma+\text { fix } x \cdot M: \tau^{\prime} \rightarrow \sigma} \\
& T(\Gamma) \cup\left\{\tau_{1}^{\prime} \ldots \tau_{m}^{\prime}\right\} \subseteq T^{+} \\
& \frac{\Gamma, x: \tau^{\prime} \rightarrow \tilde{\sigma} \vdash M: \tau^{\prime} \rightarrow \dot{\sigma}^{+}}{\Gamma \vdash \text { fix } x \cdot M: \tau^{\prime} \rightarrow \dot{\sigma}^{+}} \\
& \frac{\Gamma \vdash M: \tau, \tau \leq \tau^{\prime}}{\Gamma \vdash M: \tau^{\prime}} \\
& \overline{\Gamma \vdash f_{i}^{\sigma}:[\tilde{\sigma} / t] \tau_{i, 1} \rightarrow \cdots \rightarrow[\tilde{\sigma} / t] \tau_{i, n_{i}} \rightarrow \dot{\sigma}^{F}} \\
& \begin{array}{c}
\overline{\Gamma \vdash \text { case }^{\sigma}: \dot{\sigma}^{+} \rightarrow\left([\tilde{\sigma} / t] \tau_{1} \rightarrow \tau\right) \rightarrow \cdots\left([\tilde{\sigma} / t] \tau_{k} \rightarrow \tau\right) \rightarrow \tau} \\
\overline{\tau \leq \tau} \overline{\sigma \leq \check{\sigma}^{+}} \overline{\sigma \leq \dot{\sigma}} \overline{\tilde{\sigma}^{+} \leq \check{\sigma}} \frac{\tau_{2} \leq \tau_{1} \tau_{1}^{\prime} \leq \tau_{2}^{\prime}}{\tau_{1} \rightarrow \tau_{1}^{\prime} \leq \tau_{2} \rightarrow \tau_{2}^{\prime}}
\end{array}
\end{aligned}
$$

We give some motivation and intuition for these rules. In the first rule, the condition $M \downarrow x$ is replaced by the typing judgment $\Gamma, x: \tau \rightarrow \check{\sigma} \vdash M: \tau \rightarrow \check{\sigma}^{+}$. The second rule for fix is used to type nested fixpoints as in example 2(6). In the rules for fix, the side conditions $T(\Gamma) \cup\left\{\tau_{1}^{\prime} \ldots \tau_{k}^{\prime}\right\} \subseteq T$ and $T(\Gamma) \cup$ $\left\{\tau_{1}^{\prime} \ldots \tau_{k}^{\prime}\right\} \subseteq T^{+}$guarantee independence and anti-monotonicity, respectively, of the type interpretation with respect to the ordinal parameter. 
The additional rule for the constructors $f_{i}$ is needed to introduce terms of type $\check{\sigma}^{+}$. Note that in this way we overload the constructors $f_{i}$ by giving them two related types (but incomparable with respect to subtyping). There is also a related rule which overloads the destructor case.

The following rules just state the subtyping relations between $\sigma, \check{\sigma}$, and $\check{\sigma}^{+}$, and the way this relation is lifted higher-order. The obvious transitivity rule for the subtyping relation $\leq$ can be derived. Types with the relation $\leq$ form a quite simple partial order. In particular, if $R=\leq U \leq \leq^{-1}$ then $\left\{\tau^{\prime} \mid \tau \bar{R}^{*} \tau^{\prime}\right\}$ is finite. We state some basic properties of the typing system.

Lemma 5. (1) Exchange. If $\Gamma, x: \tau_{1}, y: \tau_{2}, \Gamma^{\prime} \vdash M: \tau$ then $\Gamma, y: \tau_{2}, x:$ $\tau_{1}, \Gamma^{\prime} \vdash M: \tau$ (with a proof of the same depth).

(2) Remove. If $\Gamma, x: \tau^{\prime} \vdash M: \tau$ and $x \notin F V(M)$, then $\Gamma \vdash M: \tau$.

(3) Weakening (restricted). If $\Gamma \vdash M: \tau, x$ fresh, and either $\tau^{\prime} \in T$ or fix does not occur in $M$ then $\Gamma, x: \tau^{\prime} \vdash M: \tau$.

(4) Transitivity. If $\leftarrow \leq \tau^{\prime}$ and $\vdash \tau^{\prime} \leq \tau^{\prime \prime}$ then $\vdash \tau \leq \tau^{\prime \prime}$.

(5) Substitution. If $\Gamma, x: \tau^{\prime} \vdash M: \tau$ and $\Gamma \vdash N: \tau^{\prime}$ then $\Gamma \vdash[N / x] M: \tau$.

The terms typable using Coquand's guard condition, are strictly contained in the terms typable in the proposed typing system (as a matter of fact, all examples in 2 (but (3) of course) can be typed). This is a consequence of the following lemma.

Lemma 6. (1) If $\Gamma, x: \tau \rightarrow \sigma \vdash M: \tau, x \notin F V(M)$, and $M$ has no occurrence of fix, then $\Gamma, x: \tau \rightarrow \dot{\sigma} \vdash M: \tau$.

(2) If $\Gamma, x: \tau \rightarrow \sigma \vdash M \downarrow_{0}^{\tau^{\prime} \rightarrow \sigma} x$ then $\Gamma, x: \tau \rightarrow \check{\sigma} \vdash M: \tau^{\prime} \rightarrow \check{\sigma}$.

(3) If $\Gamma, x: \tau \rightarrow \sigma \vdash M \downarrow_{1}^{r^{\prime} \rightarrow \sigma} x$ then $\Gamma, x: \tau \rightarrow \check{\sigma} \vdash M: \tau^{\prime} \rightarrow \dot{\sigma}^{+}$.

We parameterize the type interpretation on an ordinal $\alpha$, and we define for $\sigma=\nu t .\left(f_{1}: \tau_{1} \rightarrow t \ldots f_{k}: \tau_{k} \rightarrow t\right):$

$$
\begin{array}{ll}
\llbracket t \rrbracket_{\eta}^{\alpha}=\eta(t) & \llbracket \tau \rightarrow \tau^{\prime} \rrbracket_{\eta}^{\alpha}=\llbracket \tau \rrbracket_{\eta}^{\alpha} \rightarrow \mathbb{\llbracket} \tau^{\prime} \rrbracket_{\eta}^{\alpha} \\
\llbracket \sigma \rrbracket_{\eta}^{\alpha}=g f p\left(\mathcal{F}_{\sigma, \eta, \alpha}\right) & \mathcal{F}_{\sigma, \eta, \alpha}(A)=\Sigma_{i=1 \ldots k}\left(\Pi_{j=1 \ldots n_{i}} \llbracket \sigma \rrbracket_{\eta[A / t}^{\alpha}\right) \\
\llbracket \tilde{\sigma} \rrbracket_{\eta}^{\alpha}=\mathcal{F}_{\sigma, \eta, \alpha}^{\alpha} & \llbracket \dot{\sigma}^{+} \rrbracket_{\eta}^{\alpha}=\mathcal{F}_{\sigma, \eta, \alpha}^{\alpha+1} .
\end{array}
$$

Remark. If $\tau \in T$ then $\llbracket \tau \rrbracket_{\eta}^{\alpha}$ does not depend on $\alpha$. In particular, if $\check{\sigma} \in T^{\prime}$ or $\check{\sigma}^{+} \in T^{\prime}$ then $\sigma \in T$ and therefore $\mathcal{F}_{\sigma, \eta, \alpha}=\mathcal{F}_{\sigma, \eta}$. If $\tau \in T^{+}$and $\alpha \leq \alpha^{\prime}$ then $\llbracket \tau \rrbracket_{\eta}^{\alpha} \supseteq \llbracket \tau \rrbracket_{\eta}^{\alpha^{\prime}}$, since the types of the shape $\check{\sigma}$ and $\check{\sigma}^{+}$occur in positive position.

Let us now consider the soundness of the typing rules. If $P$ is a pure $\lambda$-term, we write $x_{1}: \tau_{1} \ldots x_{n}: \tau_{n} \vDash P: \tau$ if

$$
\forall \alpha, \eta\left(\left(\forall i \in\{1 \ldots n\} d_{i} \llbracket \tau_{i} \rrbracket_{\eta}^{\alpha} d_{i}^{\prime}\right) \Rightarrow\left(\llbracket P \rrbracket[\mathrm{d} / \mathbf{x}] \llbracket \tau \rrbracket_{\eta}^{\alpha} \llbracket P \rrbracket\left[\mathbf{d}^{\prime} / \mathbf{x}\right]\right)\right) .
$$

Proposition 7 soundness. If $\Gamma \vdash M: \tau$ then $\Gamma \vDash \operatorname{er}(M): \tau$. 
It follows from proposition 7 that: $\vdash M: \tau \Rightarrow \llbracket \operatorname{er}(M) \rrbracket \in|\llbracket \tau \rrbracket|$. This result justifies the interpretation of a typed term as the equivalence class of its erasure (it is straightforward to adapt this interpretation to take into account contexts and environments). Thus, if $\vdash M: \tau$, then we set $\llbracket M \rrbracket=[\llbracket \operatorname{er}(M) \rrbracket]_{[\tau]}$.

Clearly, there is a trade-off between power and simplicity/decidability of the type system. Our contribution here is to offer a framework in which this tradeoff can be studied, and to extract from it one possible type system. We will see in section 4 that this 'experimental' type system has some desirable syntactic properties, and we will discuss its relationships with Gimenez's system. We hint here, by example, to limits and possible extensions of the system.

(1) The following two definitions 'make sense' but are not typable. Here we work with the type of infinite streams $\sigma=\nu t$.(cons : $o \rightarrow t \rightarrow t$ ):

- If $x$ is a stream of numerals we denote with $x_{i}$ its $i^{\text {th }}$ element. We define a function $F$ such that $F(x)_{i}=\left(\operatorname{suc}^{\left(2^{i}\right)} x_{i}\right)$, for $i \in \omega$ :

$$
F \equiv \operatorname{fix} f \cdot \lambda x \cdot \operatorname{cons}^{\sigma}(\operatorname{suc}(h d x))(f(f(t l x))) \text {. }
$$

- A 'constant' definition which determines the infinite stream of 0 's.

$$
\text { fix } \left.x \text {. } \text { case }^{\sigma} x\left(\lambda n . \lambda y \text {. (fix } x^{\prime} . \text { cons }^{\sigma} 0 x^{\prime}\right)\right) \text {. }
$$

(2) We can soundly generalize the two rules for fix as follows:

$$
\begin{array}{cc}
T(\Gamma) \cup\left\{\tau_{1}^{\prime} \ldots \tau_{m}^{\prime}\right\} \subseteq T \text { pos }\left(t, \tau_{i}^{\prime}\right) & T(\Gamma) \cup\left\{\tau_{1}^{\prime} \ldots \tau_{m}^{\prime}\right\} \subseteq T^{+} \text {pos }\left(t, \tau_{i}^{\prime}\right) \\
\Gamma, x:[\sigma / t]\left(\tau^{\prime} \rightarrow t\right) \vdash M:[\sigma / t]\left(\tau^{\prime} \rightarrow t\right) & \Gamma,[\tilde{\sigma} / t]\left(\tau^{\prime} \rightarrow t\right) \vdash M:\left[\tilde{\sigma}^{+} / t\right]\left(\tau^{\prime} \rightarrow t\right) \\
\frac{\Gamma, x:[\tilde{\sigma} / t]\left(\tau^{\prime} \rightarrow t\right) \vdash M:\left[\tilde{\sigma}^{+} / t\right]\left(\tau^{\prime} \rightarrow t\right)}{\Gamma+\operatorname{fix} x . M:[\tilde{\sigma} / t]\left(\tau^{\prime} \rightarrow t\right)} & \frac{\Gamma \cdot M:[\sigma / t]\left(\tau^{\prime} \rightarrow t\right)}{\Gamma \vdash \operatorname{fix} x \cdot t)}
\end{array}
$$

where $\tilde{\sigma} \in\left\{\tilde{\sigma}, \tilde{\sigma}^{+}\right\}$. These rules are particularly powerful and will be analysed in a forthcoming paper. For instance, they can be used to type: the representation of a sequential circuit as a function over streams of booleans (we found the rules trying this example), the example (11) above, and a tail append function.

(3) One may consider the extension of the type system with a finite or infinite hierarchy of approximating types, say: $\sigma \leq \cdots \leq \dot{\sigma}^{+++} \leq \dot{\sigma}^{++} \leq \dot{\sigma}^{+} \leq \dot{\sigma}$.

Next we turn to equations. We say that an equation $M=N: \tau$ is valid in the per interpretation, if

$$
\forall \Gamma(\Gamma \vdash M: \tau \text { and } \Gamma \vdash N: \tau \Rightarrow \Gamma \vDash M=N: \tau)
$$

where $x_{1}: \tau_{1} \ldots x_{n}: \tau_{n} \vDash M=N: \tau$, if

$$
\forall \alpha, \eta\left(\left(\forall i \in\{1 \ldots n\} d_{i} \llbracket \tau_{i} \rrbracket_{\eta}^{\alpha} d_{i}^{\prime}\right) \Rightarrow \llbracket \operatorname{er}(M) \rrbracket[\mathrm{d} / \mathrm{x}] \llbracket \tau \rrbracket_{\eta}^{\alpha} \llbracket \operatorname{er}(N) \rrbracket\left[\mathrm{d}^{\prime} / \mathrm{x}\right]\right) .
$$

Reasoning at the level of erasures, it is easy to derive some valid equations.

Proposition 8 valid equations. The following equations are valid in the per interpretation:

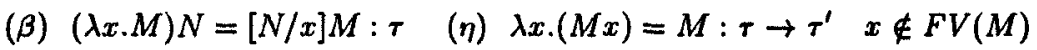

$$
\begin{aligned}
& \text { (case) }\left(\text { case }^{\sigma}\left(f_{i}^{\sigma} M_{1} \ldots M_{n_{i}}\right) N\right)=N_{i} M_{1} \ldots M_{n_{i}}: \tau \\
& \left(\text { case }_{n}\right)\left(\text { case }^{\sigma} x f_{1}^{\sigma} \ldots f_{k}^{\sigma}\right)=x: \sigma \text { (fix) fix } x . M=[\text { fix } x . M / x] M: \tau \rightarrow \sigma \text {. }
\end{aligned}
$$


The following proposition introduces an important principle to prove the equality of terms of co-inductive type.

Proposition 9 unique fixed point. Suppose $\Gamma \vdash N: \tau \rightarrow \sigma, \Gamma \vdash N^{\prime}: \tau \rightarrow$ $\sigma, \Gamma, x: \tau \rightarrow \breve{\sigma} \vdash M: \tau \rightarrow \check{\sigma}^{+}$, and $T(\Gamma) \cup\{\tau\} \subseteq T$. Then $\Gamma \models[N / x] M=N:$ $\tau \rightarrow \sigma$ and $\Gamma \vDash\left[N^{\prime} / x\right] M=N^{\prime}: \tau \rightarrow \sigma$ implies $\Gamma \vDash N=N^{\prime}: \tau \rightarrow \sigma$.

Proposition 9 resembles Banach's theorem: contractive functions have a unique fixed point (in our case, 'contractive' is replaced by 'guarded'). Combining with unfolding (fix), one can then prove equivalences such as (cf. [18]):

$$
\text { fix } x \text {.cons } n \text { (cons } n x)=\text { fix } x \text {.cons } n x \text {. }
$$

An interesting question is whether the interpretation identifies as many closed terms of co-inductive type as possible. We consider this question for the type of streams of numerals $\sigma=\nu t$. (cons : $o \rightarrow t \rightarrow t$ ) (cf. example 2) and leave the generalization to a following paper. Suppose that for $M, N$ closed terms of type $o$ we have:

$$
M=N: o \text { iff } \llbracket M \rrbracket=\llbracket N \rrbracket
$$

where the left equality denotes conversion. We define a simulation relation $\sim \omega$ over the closed terms of type $o$, say $\Lambda_{\sigma}^{0}$ as $\sim^{\omega}=\bigcap_{n<\omega} \sim^{n}$, where:

$$
\sim^{0}=\Lambda_{\sigma}^{0} \times \Lambda_{o}^{0} \quad \sim^{n+1}=\left\{(M, N) \mid\left(h d M=h d N \text { and }(t l M, t l N) \in \sim^{n}\right)\right\} .
$$

Equivalently, we can characterize $\sim^{\omega}$ as:

$$
M \sim^{\omega} N \text { iff } \forall n \in \omega h d\left(t l^{n} M\right)=h d\left(t l^{n} N\right) .
$$

Clearly $\sim^{\omega}$ is the largest (sensible) equivalence we can expect on $\Lambda_{\sigma}^{0}$. We can show that this equivalence is precisely that induced by the per's interpretation.

Proposition 10. Let $M, N \in \Lambda_{\sigma}^{0}$. Then $M \sim^{w} N$ iff $\llbracket M \rrbracket=\llbracket N \rrbracket$.

\section{Reduction}

It is easy to see that the equality induced by the per's interpretation on coinductive types is in general undecidable (E.g., let the $n^{\text {th }}$ element of a stream witness the termination of a Turing machine after $n$ steps). In the presence of dependent types (like in the Calculus of Constructions), it is imperative to have a theory of conversion which is decidable. Thus the approach is to: (i) Consider a weaker (but decidable) notion of conversion on terms, and (ii) Define in the logical system a notion of term equivalence which captures the intended meaning, e.g., using a notion of simulation as in (13). A standard way to achieve decidability for an equational theory is to exhibit a rewriting system which is confluent and terminating. In order to achieve termination, the unfolding of fixpoints has to be restricted somehow. Gimenez has proposed a solution in which fix is unfolded only under a case. Intuitively, fix is considered as an additional constructor 
which can be simplified only when it meets the corresponding destructor. ${ }^{3}$ In the following we will simplify the matter by ignoring the extensional rules:

$$
\begin{array}{ll}
(\lambda x . M) N & \rightarrow[N / x] M \\
\text { case }^{\sigma}\left(f_{i}^{\sigma} \mathrm{M}\right) \mathrm{N} & \rightarrow N_{i} \mathrm{M} \\
\text { case }^{\sigma}((\text { fix } x . M) \mathrm{M}) \mathrm{N} & \rightarrow \text { case }^{\sigma}(([\mathrm{fix} x . M / x] M) \mathrm{M}) \mathrm{N} .
\end{array}
$$

We also denote with $\rightarrow$ the compatible closure of the rules above. It is easily seen that the resulting rewriting system is locally confluent. Subject reduction is stated as follows.

Proposition 11. If $\Gamma \vdash M: \tau$ and $M \rightarrow M^{\prime}$ then $\Gamma \vdash M^{\prime}: \tau$.

The strong normalization proof is based on an interpretation of types as reducibility candidates. We outline the construction (which is quite similar to the one for per's) by assuming that there is just one ground type $o$ and one co-inductive type $\sigma=\nu t$. (cons : $0 \rightarrow t \rightarrow t$ ). Let $S N$ be the set of strongly normalizing terms. We say that a term is not neutral if it has the shape (we omit the type labels on cons and case):

$\lambda x . M$, cons $M,($ fix $x . M) M$, case, case(cons $\left.M_{1} M_{2}\right)$, case((fix $\left.\left.x . M\right) M\right)$.

We note a fundamental property of neutral terms.

Lemma 12. If $M$ is neutral, then for any term $N, M N$ and case $M N$ are neutral, and they are not redexes.

Therefore a reduction of $M N$ (or case $M N$ ) is either a reduction of $M$ or a reduction of $N$. Following closely [10], we define the collection of reducibility candidates.

Definition 13. The set of terms $X$ belongs to the collection $R C$ of reducibility candidates if: $\left(C_{1}\right) X \subseteq S N$. $\left(C_{2}\right)$ If $M \in X$ and $M \rightarrow M^{\prime}$ then $M^{\prime} \in X .\left(C_{3}\right)$ If $M$ is neutral and $\forall M^{\prime}\left(M \rightarrow M^{\prime} \Rightarrow M^{\prime} \in X\right)$ then $M \in X$.

The following are standard properties of reducibility candidates (but for $\left(P_{5}\right)$ and $\left(P_{6}\right)$ which mutatis mutandis appear in [8]):

Proposition 14. The set $R C$ enjoys the following properties:

$\left(P_{1}\right) S N \in R C$.

$\left(P_{2}\right)$ If $X \in R C$ then $x \in X$. Hence $X \neq \emptyset$.

$\left(P_{3}\right)$ If $X, Y \in R C$ then $X \rightarrow Y=\{M \mid \forall N \in X(M N \in Y)\} \in R C$.

$\left(P_{4}\right)$ If $\forall i \in I X_{i} \in R C$ then $\bigcap_{i \in I} X_{i} \in R C$.

$\left(P_{5}\right)$ If $X \in R C$ then

$$
\mathcal{N}(X)=\{M \mid \forall Y \in R C \forall P \in S N \rightarrow X \rightarrow Y \text { case } M P \in Y\} \in R C .
$$

$\left(P_{6}\right)$ If $X \subseteq X^{\prime}$ then $\mathcal{N}(X) \subseteq \mathcal{N}\left(X^{\prime}\right)$.

\footnotetext{
${ }^{3}$ Another possible approach, is to stop unfolding under a constructor. However this leads to a non-confluent system (exactly as in a 'weak' $\lambda$-calculus where reduction stops at $\lambda$ 's).
} 
We can then define the type interpretation which is (again) parameterized on an ordinal $\alpha$ (of course, we take $\mathcal{N}^{0}=S N$ ):

$$
\begin{aligned}
\llbracket o \rrbracket^{\alpha}=S N & \llbracket \tau \rightarrow \tau^{\prime} \mathbb{1}^{\alpha}=\llbracket \tau \rrbracket^{\alpha} \rightarrow \llbracket \tau^{\prime} \rrbracket^{\alpha} \\
\llbracket \sigma \rrbracket^{\alpha}=g f p(\mathcal{N}) & \llbracket \check{\sigma} \rrbracket^{\alpha}=\mathcal{N}^{\alpha} \quad \llbracket \check{\sigma}^{+} \rrbracket^{\alpha}=\mathcal{N}^{\alpha+1} .
\end{aligned}
$$

We define $x_{1}: \tau_{1} \ldots x_{n}: \tau_{n} \vDash_{R C} M: \tau$ if $\forall \alpha\left(\left(\forall i \in\{1 \ldots n\} P_{i} \in \llbracket\left[\tau_{i} \rrbracket^{\alpha}\right) \Rightarrow\right.\right.$ $\left.\left[P_{1} / x_{1} \ldots P_{n} / x_{n}\right] M \in \mathbb{[} r \mathbb{1}^{\alpha}\right)$. We can then state the following result from which strong normalization immediately follows by taking $P_{i} \equiv x_{i}$.

Proposition 15 strong normalization. If $\Gamma \vdash M: \tau$ then $\Gamma \vDash_{R C} M: \tau$.

Remark. From these results, we can conclude that it is always better to normalize the body $M$ of a recursive definition fix $x . M$, before checking the guard condition, e.g., consider: $M \equiv\left(\lambda z\right.$.case $\left.z\left(\lambda n . \lambda z^{\prime} . z^{\prime}\right)\right)$ (cons $n$ (cons $\left.n x\right)$ ). This term cannot be typed, but if $M^{\prime}$ is the normal form of $M$ then fix $x . M^{\prime}$ can be typed.

In his thesis, Gimenez has studied an extension of the calculus of constructions with the co-inductive type of finite and infinite streams (cf. example 2(5)). In the Coq system, the user can actually introduce other co-inductive types. Among the examples of co-inductive type considered in this paper, the type in example 1(3) is the only one which is rejected. The reason is that Coq relies on a stricter notion of positivity to avoid some consistency problems which arise at higher-order types [9]. It should be noted that Coq implementation of coinductive types was developed before the type theory was settled, and cannot be considered as a faithful implementation of it.

We sketch a semantic reconstruction of Gimenez's system. In the interpretation studied in section 3, all approximating types are assigned the same ordinal. We might consider a more liberal system in which different ordinals can be assigned to different approximating types. However, to express the guard condition, we still need a linguistic mechanism to say in which cases the ordinal assignment really has to be the same. Following this intuition, we label the approximating types with the intention to assign an ordinal to each label. As before, we restrict our attention to the type of infinite streams, say $\sigma$ with constructor cons : $o \rightarrow \sigma \rightarrow \sigma$. The collection of types is then defined as follows:

$$
\tau::=o|\sigma| \sigma^{x}\left|\sigma^{x+1}\right|(\tau \rightarrow \tau) \text {. }
$$

Roughly, we replace the type $\check{\sigma}$ with the types $\sigma^{x}$ and the type $\check{\sigma}^{+}$with the types $\sigma^{x+1}$, where $x$ is a label which we take for convenience as ranging over the set of term variables $x, y, \ldots$ (any other infinite set would do). More precisely, if $h$ denotes an assignment from variables to ordinals then we define a type interpretation parametric in $h$.

$$
\begin{aligned}
& \llbracket o \rrbracket_{h}=O \text { (for some chosen per } O \text { ) } \llbracket \tau \rightarrow \tau^{\prime} \rrbracket_{h}=\llbracket \tau \rrbracket_{h} \rightarrow \llbracket \tau^{\prime} \rrbracket_{h} \\
& \llbracket \sigma \rrbracket_{h}=g f p(\mathcal{F}) \\
& \llbracket \sigma^{x} \rrbracket_{h}=\mathcal{F}^{h(x)} \\
& \mathcal{F}(A) \quad=O \times A \\
& \llbracket \sigma^{x+1} \rrbracket_{h}=\mathcal{F}^{h(x)+1} \text {. }
\end{aligned}
$$


If $P$ is a pure $\lambda$-term, we write $x_{1}: \tau_{1} \ldots x_{n}: \tau_{n} \vDash P: \tau$ if

$$
\forall h\left(\left(\forall i \in\{1 \ldots n\} d_{i} \llbracket \tau_{i} \rrbracket_{h} d_{i}^{\prime}\right) \Rightarrow\left(\llbracket P \rrbracket[\mathbf{d} / \mathbf{x}] \llbracket \tau \rrbracket_{h} \llbracket P \rrbracket\left[\mathbf{d}^{\prime} / \mathbf{x}\right]\right)\right) .
$$

We now turn to syntax. Let $\operatorname{var}(r)$ be the set of variables which occur in the type $\tau$. If $\Gamma$ is a context, we also define $\operatorname{var}(\Gamma)=\bigcup\{\operatorname{var}(\tau) \mid x: \tau \in \Gamma\}$. If $x$ is a variable, we define $T^{+}(x)$ as the set of types such that all subtypes of the form $\sigma^{x}$ or $\sigma^{x+1}$ occur in positive position. Following the interpretation above, the typing rules for, e.g., fix can be formulated as follows, where $\tau^{\prime} \rightarrow \sigma^{u} \equiv \tau_{1}^{\prime} \rightarrow$ $\cdots \rightarrow \tau_{m}^{\prime} \rightarrow \sigma^{u}, m \geq 0, u$ can be a label or nothing.

$$
\begin{array}{cc}
x \notin \operatorname{var}(\Gamma) \cup \bigcup\left\{\operatorname{var}\left(\tau_{i}^{\prime}\right) \mid i=1 \ldots m\right\} & T(\Gamma) \cup\left\{\tau_{i}^{\prime} \mid i=1 \ldots m\right\} \subseteq T^{+}(y) \\
\Gamma, x: \tau^{\prime} \rightarrow \sigma \vdash M: \tau^{\prime} \rightarrow \sigma & \Gamma, x: \tau^{\prime} \rightarrow \sigma^{y} \vdash M: \tau^{\prime} \rightarrow \sigma^{y+1} \\
\Gamma, x: \tau^{\prime} \rightarrow \sigma^{x} \vdash M: \tau^{\prime} \rightarrow \sigma^{x+1} & \frac{\Gamma \vdash \text { fix } x . M: \tau^{\prime} \rightarrow \sigma^{y+1}}{\Gamma \vdash \text { fix } x . M: \tau^{\prime} \rightarrow \sigma}
\end{array}
$$

Soundness can be proved as for proposition 7. When Gimenez's system is considered in a simply-typed framework, the following differences appear with respect to the system with labelled types (ignoring some minor notational conventions): (1) Gimenez's typing system is presented in a 'Church' style. More precisely, the variables bound by $\lambda$ and fix carry a type, and this type is used to constraint (in the usual way) the application of the related typing rules. (2) The subtyping rule for functional types $\tau \rightarrow \tau^{\prime}$ is missing. (3) The second rule for typing recursive definitions is missing.

Obviously these differences imply that one can give less types to a term in Gimenez's system than in our system. To be fair, one has to notice that the presentation as a Church system and the absence of subtyping at higher-types is essentially justified by the complexity of the calculus of constructions, and by the desire to avoid too many complications at once. On the other hand, the lack of the second rule for fix is, in our opinion, a genuine difference, which moreover has an impact in practice, as the rule is needed to type nested recursive definitions as that of example 2(6) and can be further generalized as shown in (12). A question which should be raised is whether the system with type labels is better in practice than the simpler system without type labels. So far, we could not find any 'natural' example suggesting a positive answer.

Acknowledgement The first author would like to thank Eduardo Gimenez for providing the simply typed formulation of his system and explaining its motivations, and Alexandra Bac for a number of discussions on the type system presented here.

\section{References}

1. R. Amadio and S. Coupet-Grimal. Analysis of a guard condition in type theory (preliminary report). Technical Report TR 1997.245. Also appeared as RRINRIA 3300, Université de Provence (LIM), 1997. Available at http://protis.univmrs.fr/ amadio. 
2. H. Barendregt. The lambda calculus; its syntax and semantics. North-Holland, 1984.

3. Coq-project. The Coq proof assistant reference manual. Available at http://pauillac.inria.fr/coq, 1996.

4. T. Coquand. Infinite objects in type theory. In Types for proofs and programs, Springer Lect. Notes in Comp. Sci. 806, 1993.

5. T. Coquand and G. Huet. A calculus of constructions. Information and Computation, 76:95-120, 1988.

6. S. Coupet-Grimal and L. Jakubiec. Coq and hardware verification: a case study. In Proc. TPHOL, Springer Lect. Notes in Comp. Sci. 1125, 1996.

7. H. Geuvers. Inductive and coinductive types with iteration and recursion. In Proc. of Workshop on types for proofs and programs, Nordström et al. (eds.), pages 193217, 1992. Available electronically.

8. E. Gimenez. Un calcul de constructions infinies et son application à la vérification de systèmes communicants. PhD thesis, ENS Lyon, 1996.

9. E. Gimenez. Personal communication. October 1997.

10. J.-Y. Girard, Y.Lafont, and P. Taylor. Proofs and Types. Cambridge University Press, 1989.

11. F. Leclerc and C. Paulin-Morhing. Programming with streams in Coq. A case study: the sieve of Eratosthenes. In Proc. TYPES, Springer Lect. Notes in Comp. Sci. 806, 1993.

12. R. Loader. Equational theories for inductive types. Annals of Pure and Applied Logic, 84:175-218, 1997.

13. G. Longo and E. Moggi. Constructive natural deduction and its modest interpretation. Mathematical Structures in Computer Science, 1:215-254, 1992.

14. N. Mendler. Recursive types and type constraints in second-order lambda calculus. In Proc. IEEE Logic in Comp. Sci., 1987.

15. M. Nesi. A formalization of the process algebra CCS in higher order logic. Technical Report 278, Computer Laboratory, University of Cambridge, December 1992.

16. L. Paulson. Mechanizing coinduction and corecursion in higher-order logic. J. of Logic and Computation, 7(2):175-204, 1997.

17. C. Raffalli. L'arithmétique fonctionnelle du second ordre avec point fixes. PhD thesis, Université Paris VII, 1994.

18. A. Salomaa. Two complete systems for the algebra of complete events. Journal of the ACM, 13-1, 1966.

19. D. Scott. Data types as lattices. SIAM J. of Computing, 5:522-587, 1976.

20. M. Tatsuta. Realizability interpretation of coinductive definitions and program synthesis with streams. Theoretical Computer Science, 122:119-136, 1994.

21. S. Thompson. Haskell. The craft of functional programming. Addison-Wesley, 1996. 